\title{
THREE-DIMENSIONAL LASER IMAGING BASED ON A PHOTON- COUNTING AVALANCHE PHOTODIODE ARRAY
}

\author{
Peng Ge ${ }^{1,2^{*}}$, Cong Chen ${ }^{1,2}$, Zhen Shang ${ }^{1,2}$, Yanen Fan ${ }^{1,2}$, Jingjing Guo ${ }^{1,2}$, Zibo Zhuang $^{3}$, Jialong Ge ${ }^{1,2}$ \\ ${ }^{I}$ East China Research Institute of Electronic Engineering, Hefei 230088, Anhui, China \\ ${ }^{2}$ Key Laboratory of Aperture Array and Space Application, Hefei 230088, Anhui, China \\ ${ }^{3}$ Civil Aviation University of China, Tianjin 300300, China \\ *Email: gepeng_cas@qq.com
}

\begin{abstract}
Photon-counting detector array is very desired for high-resolution laser imaging based on direct time-of-flight measurement. Such systems have potential applications in remote sensing with long distances. We will perform three-dimensional imaging using a large InGaAs Geiger-mode avalanche photodiode array which has singlephoton sensitivity. To improve the image quality with only a few photon detections, the photon counting imaging process is analyzed and a regularization method based on pixel spatial correlation is employed for image reconstruction. The performance of the method is compared with that of conventional maximum likelihood estimation on intensity and range image reconstructions of a building about six hundred meters away.
\end{abstract}

\section{INTRODUCTION}

Laser radar can achieve much higher angular resolution and range precision than microwave radar due to the short wavelength of laser light ${ }^{[1]}$. For imaging at a long stand-off distance with laser radar, the received reflected light is usually very low, and single-photon detectors are widely used for the weak signal detection with its unparalleled sensitivity. The transverse resolution of the imaging is obtained either by point-by-point scanning ${ }^{[2]}$ or by large detector array ${ }^{[3]}$. As the scanning way is time consuming for large imaging, detector array is much preferred which can acquire scene information in a parallel way. Currently Geiger-mode avalanche photodiode (APD) based array is the most mature and widely used single-photon detector array, and plenty of effort has been done on the development of large Geiger-mode APD array ${ }^{[4][5]}$ and its applications on three-dimensional laser imaging ${ }^{[6[7]}$.

With the help of flood illumination of short laser pulses, Geiger-mode APD array is mainly used for direct-detect three-dimensional imaging. Benefit from the single-photon sensitivity, it is always operated at a low incident photon rate per laser pulse that leads to a low detection probability for a laser pulse. Multiple pulse collection is commonly used to achieve a high detection probability ${ }^{[8][9]}$. Geiger-mode APD records a number of discrete photon detections which is different with energy measurement with traditional light detectors ${ }^{[10]}$. The discrete photon detection data cannot be deal with conventional Gaussian noise model for image reconstruction. Alternatively, Poisson noise model shall be applied. Therefore, besides the performance of Geiger-mode APD array, the image reconstruction algorithm is also important to achieve high quality imaging $^{[11][12]}$.

In this paper, we will demonstrate the threedimensional laser imaging based on a singlephoton camera which is composed of a large InGaAs Geiger-mode APD array. Compared with that made of silicon, the Geiger-mode APD made of InGaAs absorption material has good response to longer wavelengths beyond $900 \mathrm{~nm}$ that can be used for eye-safe laser imaging. We will discuss the photon counting imaging process on the Geiger-mode APD for the intensity and range estimation. Instead of using conventional pixel by pixel maximum likelihood method, a regularization method based on the spatial correlation is employed to improve the image reconstruction quality with a few photon detections.

\section{EXPERIMENTAL SETUP}

The schematic diagram of our imaging setup is illustrated in Fig. 1 It is a bistatic system using separated transmitting and receiving apertures so that the reflections from the transmitting lens will not blind the single-photon camera. For imaging at kilometer distances, a Nd:YAG microchip laser is employed to generate $500 \mathrm{ps}$ laser pulses at 
$1064 \mathrm{~nm}$ with high peak power. The laser is passively Q-switched internally and operated at a repetition rate of $1 \mathrm{kHz}$ with output pulse energy of $80 \mathrm{uJ}$ via external trigger current. For the laser cavity building time is about 150 us with a fluctuation about $200 \mathrm{~ns}$, the emitting time of each pulse is precisely measured via an auxiliary detector to synchronize the single-photon camera to start to work.

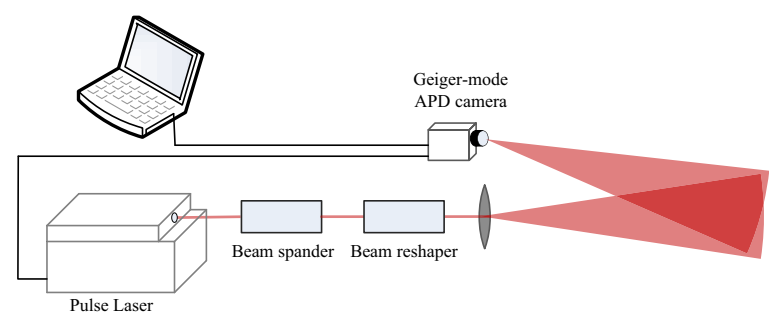

Figure 1 Schematic diagram of the imaging setup.

The laser pulses are spread to illuminate a scene of interest. However the light intensity on the laser beam cross-section is not a uniform distribution with high intensity in the center and low intensity in the edge following Gaussian distribution. As a result, the illumination efficiency is low and the received light intensities on detectors have large spatial difference. A beam shaper, pishaper_1064_6_6, is employed to transform the beam spatial intensity from Gaussian distribution to uniform distribution. As the beam shaper requires a collimated input beam spot with a diameter of $6 \mathrm{~mm}$, a beam expander is placed between the laser and the shaper. The shaper has nearly $100 \%$ transforming efficiency at $1064 \mathrm{~nm}$. The output beam is then spread to flood illuminated the scene with an optical lens.

The reflected light is collected by a small telescope with $25 \mathrm{~mm}$ receiving aperture and imaged on an focal plant array (FPA) developed by CETC44. The array is fabricated with $64 \times 64$ InGaAs Geiger-mode APDs. Each APD is backilluminated with $1 \mu \mathrm{m}$ pixel diameter and $50 \mu \mathrm{m}$ pixel pitch. A microlens array is aligned and attached on the surface of the FPA to increase the fill factor to about $60 \%$. The device is cooled to $258 \mathrm{~K}$ and operated with a reverse bias voltage of $58.5 \mathrm{~V}$ for photon-counting detection. The quantum efficiency at $1064 \mathrm{~nm}$ is about $15 \%$ and the normalized dark count rate is $8 \mathrm{kHz}-20 \mathrm{kHz}$. A readout integrated circuit (ROIC) of $64 \times 64$ array reads out APD signal with $2 \mathrm{~ns}$ time resolution through a $500 \mathrm{MHz}$ clock. The device can be operated at a maximum frame rate of $20 \mathrm{kHz}$ through a CameraLink interface.

\section{IMAGE RECONSTRUCTION}

\subsection{Photon-counting Imaging Process}

With the receiving telescope, detectors on the two-dimensional array collect reflected light from the corresponding scene patches. According to the quantum nature of light, the photons measured on a detector follow a Poisson process ${ }^{[13]}$. Apart from the reflected laser light, solar background and dark counts also contribute to the process. The received photon rate from the reflected laser light is time-varied determined by laser pulse shape and scene reflection, while solar background and dark counts have constant rates. The combined process is a time inhomogeneous Poisson process ${ }^{[14]}$. The received photon rate at detector $i$ can be written as

$$
r_{i}(t)=a_{i} s\left(t-2 z_{i} / c\right)+b_{i} .
$$

Where $s(t)$ is the temporal intensity distribution of laser pulses illuminating on each scene patch, $a_{i}$ is the fraction of light collected at detector $i$, including the effects of transmitting and receiving attenuation and object reflection, $z_{i}$ is the distance between the system and scene patch $i, c$ is the speed of light and $b_{i}$ is background light rate.

In a fix dwell time, the average received photon number $v_{i}$ can be obtained by integration on $r_{i}(t)$. The actual received photon number is an integer obeying Poisson statistics with mean value of $v_{i}$. It is a probability process that the received photons create primary photoelectron on the detector, which is donated by quantum efficiency $\eta$. The detector is then fired from the primary photoelectron. In fact the firing of the detector is also a Poisson process. Considering dark count rate $d$ the firing rate can be written as

$$
\lambda_{i}(t)=\eta r_{i}(t)+d=\eta a_{i} s\left(t-2 z_{i} / c\right)+\eta b_{i}+d
$$

Once the detector is fired, subsequent photoelectron within a short time interval will be blocked due to dead time effect and inability of counting multiple pulses. For the array's ROIC 
doesn't have the capacity of counting multiply pulses during a frame, only the first photoelectron can be recorded. In other word, if background photons or dark counts fired the detector before signal photons, the signal event will not be recorded.

\subsection{Noise Censoring}

The noise censoring is performed on the time-offlight (TOF) histogram on each detector according to their temporal difference with that of signal. As the signal has an impulsive shape, the measured TOF shall span only a few time bins in the histogram. The signal's TOF distribution is mainly determined by laser pulse width and time resolution of synchronizing. In our experiment, the laser pulse width is 500ps and time resolution of synchronizing is $4 \mathrm{~ns}$, so the convolution of the two terms is about $4.03 \mathrm{~ns}$. We take three sigma confidence interval of Gaussian distribution as signal interval. As the histogram has a time bin width of $2 \mathrm{~ns}$, the signal shall span about 6 bins. To locate the signal interval, we calculate the sum value of any six adjacent time bins and take the interval with maximum value as signal interval

$$
f=\max \sum_{i=k}^{k+5} n_{i}
$$

In case of no object present in the scene path, all photon detections come from noise and the signal interval is also wrong due to noise fluctuation. To solve this problem, we calculated the average photon number in each bin, and if $f$ exceed noise fluctuation with 5 sigma we then take it as a signal. Once the signal interval is located, the other events in the histogram will be censored.

\subsection{Regularization Method}

At very low light level, the collected number of photon detections per pixel is always a few in a limited dwell time ${ }^{[15][16]}$. As in Poisson statistics the variance is equal to the observed variable, the imaging will suffer much from low statistics. In addition, it has to take into account the impact of background light and dark counts. So it is a challenge to carry out precise image reconstruction, and one effective way is using regularization method ${ }^{[17][18]}$. The regularization method takes advantages of spatial correlations in natural images, or sparse in their spatial frequencies, for smoothness. With regularization method, a cost function is created incorporating a $\log$-likelihood term and regularization term as

$$
C(x)=-\ln L(x ; n)+\lambda R(x) .
$$

Where $x$ is the image signal to be reconstructed, $L(x ; n)$ is the likelihood term based on measured dataset $n, R(n)$ is the regularization term based on spatially correlation and $\tau$ is the regularization factor to balance the two terms. The image reconstruction is then converted to the minimization of the cost function $C(x)$.

For the regularization term, a number of functions can used to calculate the sparsity. We choose the total-variation(TV) norm of image signal in wavelet-domain for smoothness. The discrete wavelet transform (DWT) derived from Daubechies's 4-tap filters is used. So the cost function in our image reconstruction is

$$
C(x)=-\ln L(x ; n)+\lambda\left\|W^{T} x\right\|_{T V}
$$

Where, $W$ is the wavelet basis. Based on the cost function, we use Poisson negative likelihood for intensity imaging reconstruction, and use Gaussian negative likelihood for range imaging reconstruction. As the problem is strictly convex, the minimizations are carries out with matlab toolkit SPRIAL-TAP which uses quadratic separable approximations ${ }^{[11]}$.

\section{RESULTS}

With the experimental setup, we attempt to obtain the imaging of a building that is about six hundred meters away as illustrated in Fig. 2(a) The captured region of the building is marked with yellow lines in the figure. The camera is operated with a frame rate of $1 \mathrm{kHz}$ that synchronized by the microchip laser. In order to reduce ambient noise, a delay of 2 us after each pulse emitting is applied on the time gate of the camera. The detector is exposure for $2 u s$ for the measurement of back-scattered laser pulses. Fig. 2(b) shows the building's intensity image after noise censoring with photon detection data of 500 pulses. The image resolution is $64 \times 64$ as the same as the single-photon detector array size, and the average photon number per pixel per pulse is about 0.09 . Fig. 2(c) shows the reconstructed intensity image with regularization method from which we can 
see the image quality is improved with smoothness. Fig. 2(d) shows the building's range image with censored photon detection data of 500 pulses through maximum likelihood estimation. with regularization method. Compared with the conventional method, the regularization method gives a much better result of three-dimensional imaging with spare photon detection data.

Fig. 2(e) shows the reconstructed range image
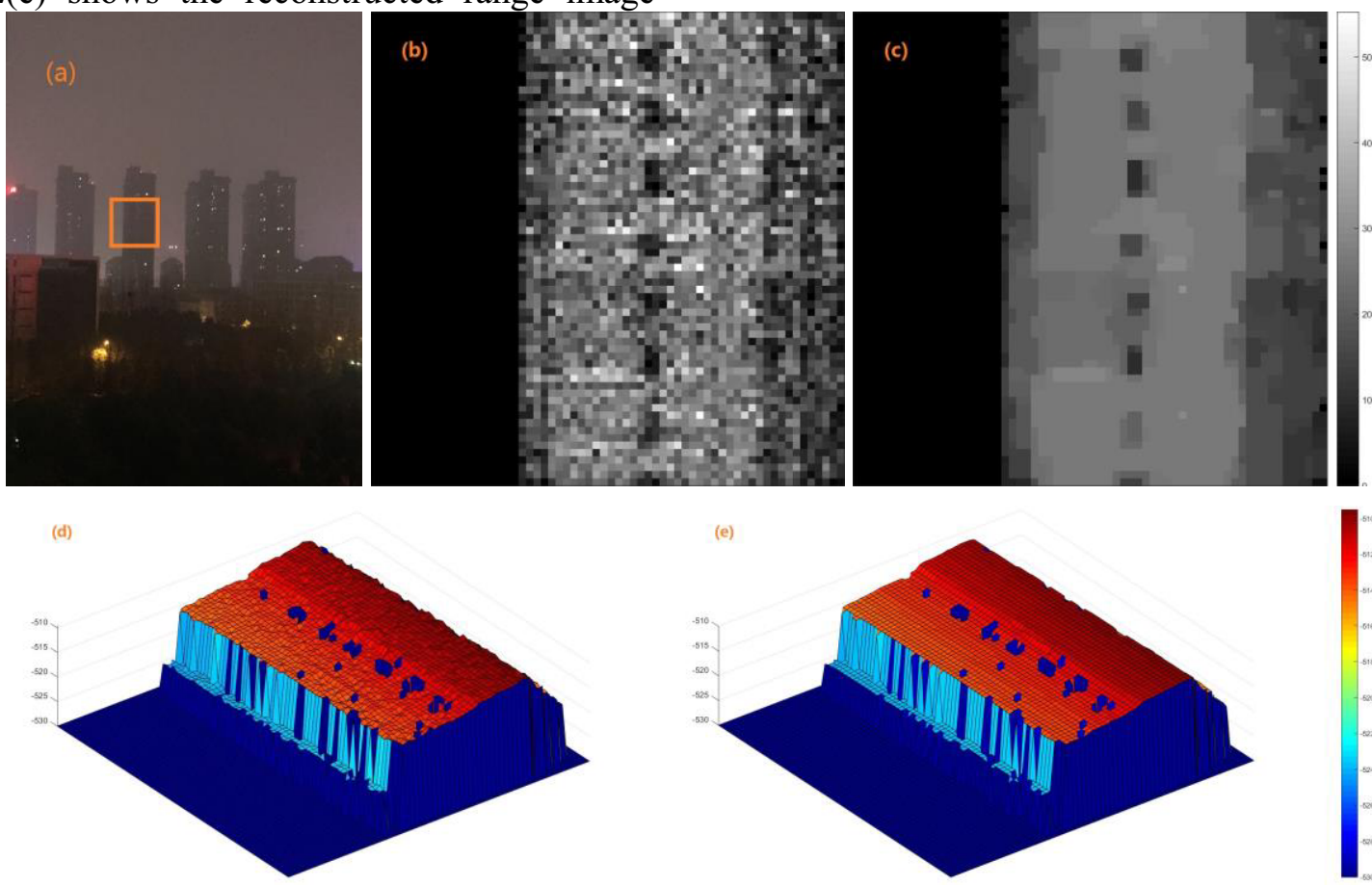

Figure 2 Photograph and photon-counting images of a building about six hundred meters away. (a) Building photograph. (b) Intensity image after noise censoring. (c) Intensity image reconstructed with regularization method. (d) Range image with maximum likelihood estimation. (e) Range image reconstructed with regularization method.

\section{ACKNOWLEDGEMENTS}

This work was supported by the CETC38 Innovation Fund and the National Natural Science Foundation of China under Grant No. U1433202.

\section{REFERENCES}

[1] P. F. McManamon, Opt. Eng. 51(6), 060901 (2012)

[2] A. McCarthy, et al. Appl. Opt. 48, 6241-6251 (2009)

[3] R. Heinrichs et al. Proc. SPIE 4377, 106-117 (2001)

[4] B. F. Aull, et al. Lincoln Lab. J. 13, 335-349 (2002)

[5] M. A. Itzler, et al. J. Mod. Opt. 58(3): 174-200 (2011)

[6] R. M. Marino, et al. Lincoln Lab. J. 15(1): 23-36 (2005)

[7] R. M. Marino, et al. Proc. SPIE 5791, 138-152 (2005)

[8] W. Becker, Springer, (2005)

[9] G. S. Buller, et al. IEEE 13, 1006-1015 (2007)

[10] P. F. McManamon, et al. Opt. Eng. 56(3): 031223 (2017)

[11] Z. T. Harmany, et al. IEEE Trans. 21, 1084-1096 (2012)
[12] L. Mertens, et al. Scientific reports, 7, 42164 (2017)

[13] D. G. Fouche, Appl. Opt. 42(27), 5388-5398 (2003)

[14] S. Kim, et al. Sensors 13(7), 8461-8489 (2013)

[15] A. C. Watts, et al. Remote Sens. 4, 1671-1692 (2012)

[16] M A. Itzlera et al. J. Mod. Opt. 58(3-4), 174-200 (2011)

[17] A. Kirmani, et al. Science 343, 58-61 (2014)

[18] D. Shin, et al. Nat. Commun. 7, 12046 (2016) 